\title{
Virtual Reality Technology Applied on Maintenance of Painted Walls of Buildings
}

\author{
Alcínia Z. Sampaio, Daniel Rosário \\ Department of Civil Engineering and Architecture, Instituto Superior Técnico, Technical University of Lisbon, Lisbon, Portugal. \\ Email: zita@civil.ist.utl.pt, derosario@gmail.com
}

Received January $1^{\text {st }}, 2012$; revised February $29^{\text {th }}, 2012$; accepted March $23^{\text {rd }}, 2012$

\begin{abstract}
In a building, the paint coating applied to interior walls conveys their aesthetic character and also performs an important function of protection. It is a construction component which is exposed to agents of deterioration related to its use, needing the regular evaluation of its state of repair. The completed model supports the performance of such periodic inspections and the monitoring of interior wall maintenance, using Virtual Reality (VR) technology. Used during an inspection visit, the application allows users to consult a database of irregularities, normally associated with paint coating, classified by the most probable causes and by recommended repair methodologies. In addition, with this model, a chromatic scale related to the degree of deterioration of the coating, defined as a function of the time between the dates of the application of the paint and the scheduled repainting, can be attributed to each element of coating monitored. This use of VR technology allows inspections and the evaluation of the degree of wear and tear of materials to be carried out in a highly direct and intuitive manner. The computer application has a positive contribution to make in the field of construction, using as it does Information Technology (IT) tools which give access to innovative technology with it capacity for interaction and visualization.
\end{abstract}

Keywords: Virtual Reality; Interactive Model; Buildings; Maintenance; Inspection

\section{Introduction}

The coating applied to building walls, naturally, performs an important aesthetic function: it is, however, essentially a protective element for the substrate on which it is applied as far as the action of environmental agents of wear and tear is concerned. The coating is fundamental to a proper overall performance of a building throughout its working life.

Materials frequently used in the coating of ordinary buildings are: paint, varnish, stone and ceramics [1]. In Portugal, where interior walls are concerned, the most commonly used coating is paint. It is a multi-purpose material, used under a variety of decorative effects, based on a widely-ranging palette of colours, patterns and textures and is easily applied on any type of surface. In addition, paint, compared to other materials, is less costly, not only as far as the product itself is concerned, but also in its application, since relatively non-specialised labour is required. Nevertheless, as deterioration is a given, maintenance is needed.

Factors such as the constant exposure of the coating to the weather, pollutants and the normal actions of housing use, linked to its natural ageing and, in some cases to the unsuitable application of systems of painting give rise to its deterioration and to the appearance of irregularities, which can negatively affect its performance as both an aesthetic and a protective element. According to Lopes [2], in normal conditions of exposure and when correctly applied a paint coating can remain unaltered for about five years. Establishing suitable maintenance strategies for this type of coating is based on the knowledge of the most frequent irregularities, the analysis of the respective causes and the study of the most suitable repair methodologies.

Currently, the management of information related to the maintenance of buildings is based on the planning of action to be taken and on the log of completed work. The capacity to visualize the process can be added through the use of three-dimensional (3D) models which, facilitate the interpretation and understanding of target elements of maintenance and of 4D models (3D + time) through which the evolution of deterioration can be visually demonstrated and understood. Furthermore, the possibility of interaction with the geometric models can be provided through the use of Virtual Technology (VR).

The work presented here is part of an on-going research project: Virtual Reality technology applied as a support tool to the planning of construction maintenance. 
PTDC/ECM/67748/2006 [3] and as such is a component of the Project focussing on the support of the maintenance activity planning with particular reference to paint coating applied to interior walls of buildings for housing.

The completed virtual model identifies the elements of the building which make up the interior wall coating so that monitoring can take place. The application is supported by a database, created for the purpose, of irregularities, their probable causes and suitable repair processes, which facilitates the inspection process. The information is recorded and associated to each monitored element, allowing subsequently, the inspection and repair activity $\log$ to be consulted, thus providing a tool for the definition of a rehabilitation strategy.

In addition, the model assigns a colour to each of the coating elements, the colours defined by the time variable, so that the evolution of the deterioration of the coating material is clearly shown through the alteration in colour. The prototype is, then, a 4D model.

The model integrates a virtual environment with an application developed in Visual Basic programming language. This allows interaction with the 3D model of buildings in such a way that it becomes possible to follow the process of monitoring the coating elements, specifically, painted interior walls, in terms of maintenance, throughout the life-cycle of the building.

\section{Maintenance}

The General Regulations for Urban Buildings (RGEU) [4] stipulates the frequency of maintenance work, stating that existing buildings must be repaired and undergo maintenance at least once every eight years with the aim of eliminating defects arising from normal wear and tear and to maintain then in good usable condition in all aspects of housing use referred to in that document.

The time-limit indicated is applicable to all elements of the buildings generally. It is clear, however, that the regulatory period is too long for some specific components and that, frequently enough, the time-limits for action are not respected. There are, too, inefficient rent policies, leading to long periods without rehabilitation, and that the prevailing culture is one of reaction on the part of the various parties involved in the maintenance process. To these aspects should also be added the defects sometimes registered during the construction of property developments, exacerbating the poor state of repair of the buildings. This gives rise to numerous irregularities which, in turn, frequently leads to inadequate safety conditions.

According to Cóias [5], the purpose of maintenance is to prolong the useful life of the building and to encourage adherence to the demands of safety and functionality, keeping in mind the specific set of conditions of each case and its budgetary considerations. Satisfactory management of this activity is carried out by putting into practice a maintenance plan which must take into consideration technical, economic, and functional aspects arising with each case.

Collen [6] points out that investment in the maintenance and rehabilitation sector in Portugal is still weak compared to that in the same sector in the construction industry in the other countries of the European Community. She makes it clear, however, on a more positive note, that some measures have already begun to be implemented here: some urban regeneration programmes have been created, legislation, which focuses on the sustainability of buildings, has been laid down, and the revision of constructive solutions has been carried out, all with the objective of guaranteeing that the maintenance of built heritage be an integral part of the construction sector.

The maintenance of buildings, then, is an activity of considerable importance within the construction industry; its contributory aspects of conservation and rehabilitation work need to be supported by correct methodologies of action, underpinned by scientific criteria and by suitable processes for the diagnosis of irregularities and the evaluation of their causes.

This paper aims to make a positive contribution to this field using the new computer technology tools of visualisation and interaction.

\section{Pathologies in Paint Coatings}

The technical document Paints, Varnishes and Painted Coatings for Civil Construction published by The National Laboratory for Civil Engineering (LNEC), defines paint as a mixture essentially made up of pigments, binder, vehicle and additives [1]. It has a pigmented, pasty composition, and when applied in a fine layer to a surface, presents, after the dispersion of volatile products, the appearance of a solid, coloured and opaque film [7].

The durability of the painted coating depends on the environment in which it is used, and on the surface it is applied to as well as the rate of deterioration of the binder in the paint. The influence of the environment is the result of the action, in conjunction or alone, of a variety of factors such as the degree of humidity, the levels of ultraviolet radiation, oxygen, ozone and alkalis, variations in temperature and of other physical or chemical agents whose effect depends considerably on the time taken to apply it [8]. When their influence is not counteracted or minimised, imperfections can arise in the coating film, such as, the appearance of defects in the layer or paint with the loss of functionality where the desired aim of the application is concerned.

These irregularities manifest themselves in various 
ways and in different degrees of severity. Based on the study made of the causes of the defects, specific methodologies for their resolution were established. Figure 1 shows common defects in painted interior walls.

The information gained from the pathological analysis of this type of coating was used to draw up a database supporting the interactive application. These data support the creation of inspection files related to the elements which are monitored in each case studied.

In order to form a user-friendly database of relatable data, groups of pathologies, shown below in Table 1, were considered. This classification provides the required automatism of access to the database and supports the presentation of synopses of the causes and repair methodology inherent in each pathology.

During the process of an on-site inspection, the user of the application can refer to the database in order to classify the abnormality being observed, consulting the list of defects, which includes, in addition to their identification, the most relevant characteristics and some of the causes that could be at the root of their development. Table 2 lists two of the irregularities from the classification: Alteration in colour.

\section{Interactive Model}

The completed application supports on-site inspections and the on-going analysis of the evolution of the degree of deterioration of the coating [10]. The following computational systems were used in its development: Auto$C A D$, in the creation of the $3 \mathrm{D}$ model of the building; EON Studio [11], for the programming of the interactiveity capacities integrated with the geometric model; Visual Basic 6 in the creation of all the windows of the application and in the establishment of links between components. All the systems were made available by the ISTAR/ DECivil [12], of the Technical University of Lisbon.

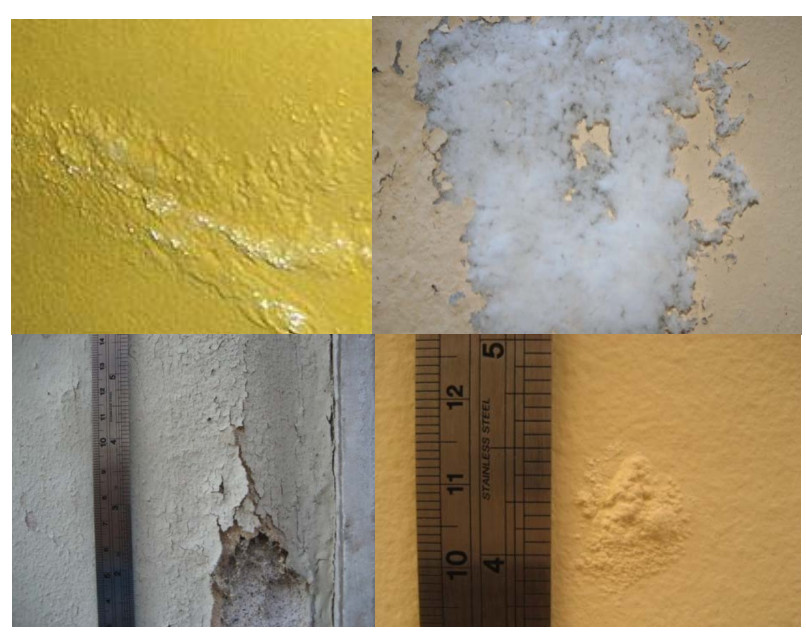

Figure 1. Swelling, efflorescence, cracking and blistering [9].

Table 1. Classification of irregularities.

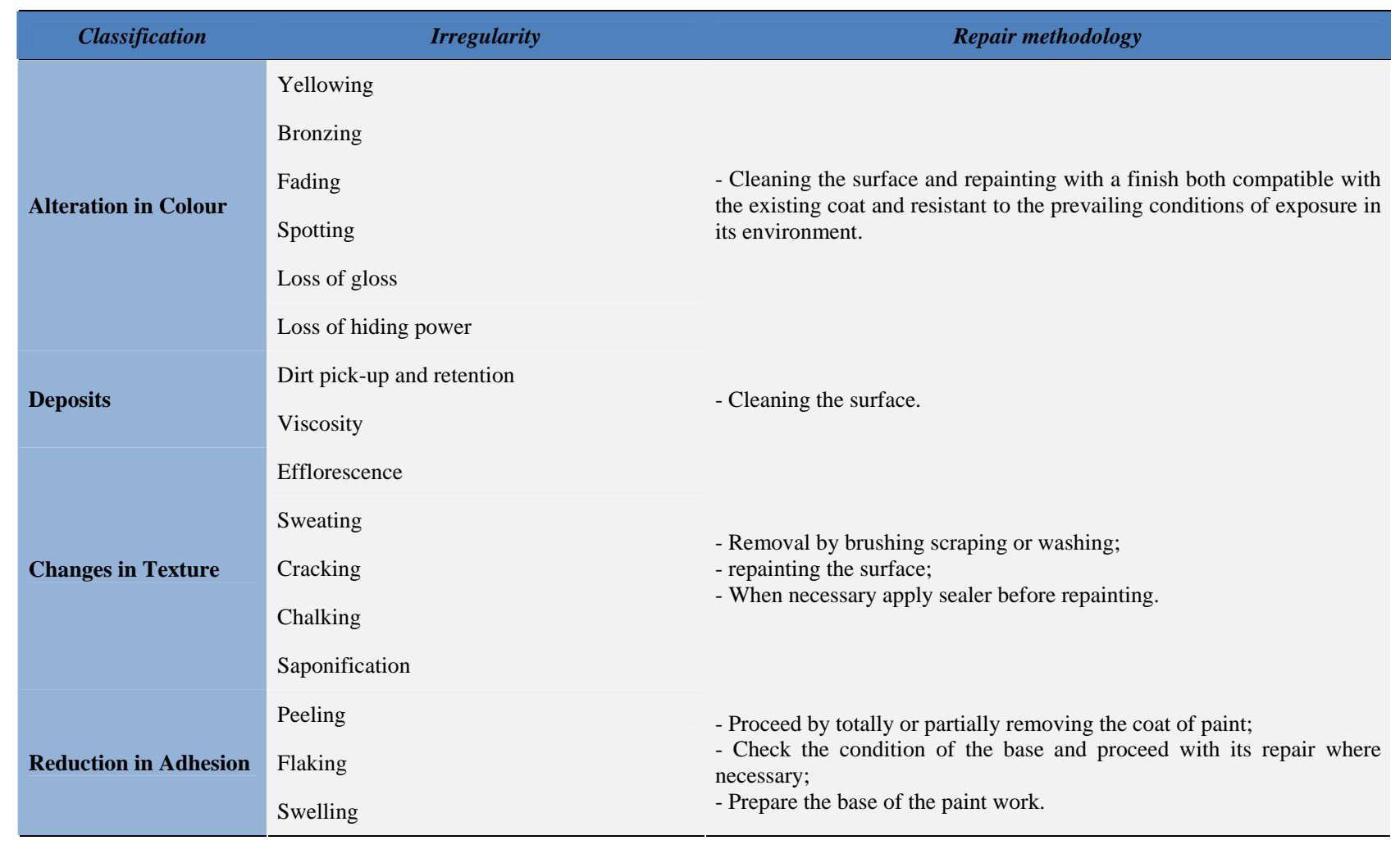


Table 2. Irregularities and causes.

\begin{tabular}{|c|c|c|}
\hline Classification & Irregularity & Characteristics and causes \\
\hline Alteration in colour & Discolouration & $\begin{array}{l}\text { - A yellow colour caused by ageing of the film of the paint or varnish; } \\
\text { - Action of environmental agents (solar radiation, temperature oxygen } \\
\text { and humidity) on the binder in the paint provoking changes in its mo- } \\
\text { lecular structure; } \\
\text { - Partial loss of colour of the film of paint coating; } \\
\text { - Action of environmental agents (solar radiation, temperature, polluted } \\
\text { atmosphere and chemically aggressive bases of application) on the binder } \\
\text { and/or the pigments of the painted coating. }\end{array}$ \\
\hline
\end{tabular}

The main interface gives access to the virtual model of the building and to the inspection and maintenance modules (highlighted in Figure 2).

The first step is to make a detailed description of the building (location, year of construction, type of structure..., Figure 3) and representative modelled elements of the interior wall coating, so that they can be monitored.

The model is manipulated in the virtual environment by using the mouse buttons (movement through the interior of the model and orientation of the camera, Figure 4). The coordinates of the observer's position and the direction of his/her point of view are associated with the element during the process of identification.

Thus, later, when an element in the database of the application is selected using the interface, the model is displayed in the visualisation window so that the target coating can be observed.

Walking through the model with the aim of accessing all the elements of the building, the user needs to be able to go up and down stairs or open doors or windows. The virtual model has been programmed, using the EON system, in such a way that these capacities are activated by positioning the cursor over the respective objects, in that way, the user is able to walk through the whole model.

Each wall surface in each of the rooms of the house is a component which has to be monitored and, therefore, to be identified. Using the model, the user must click the mouse on an element, and the message New Element is shown (highlighted in Figure 2). Associated to this selected element is the information regarding location within the house (hall, bedroom), wall type (simple internal masonry wall) and coating (paint), as shown in Figure 5.

\subsection{Making an Inspection}

Later, on an on-site inspection visit, the element to be analysed it selected interactively on the virtual model. The inspection sheet (Figure 6) is accessed by using the Inspection button which is found in the main interface (Figure 2). The data which identify the selected element are transferred to the initial data boxes on the displayed page (Figure 6).

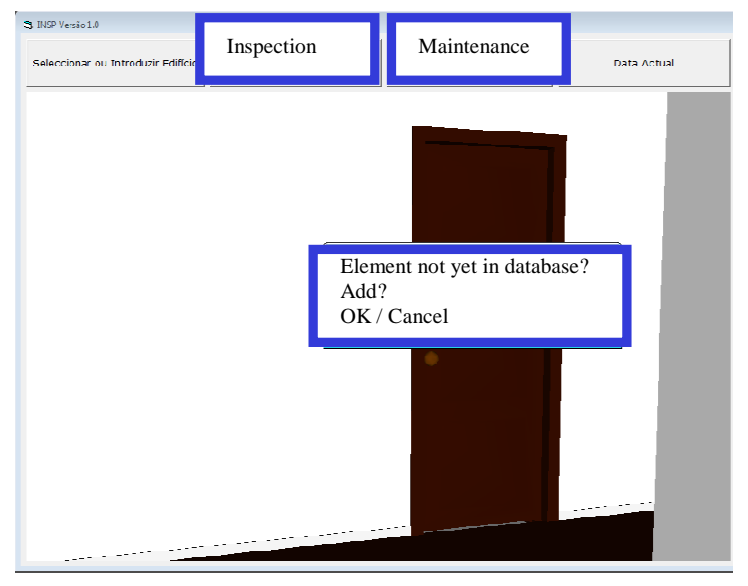

Figure 2. The main interface of the virtual application.

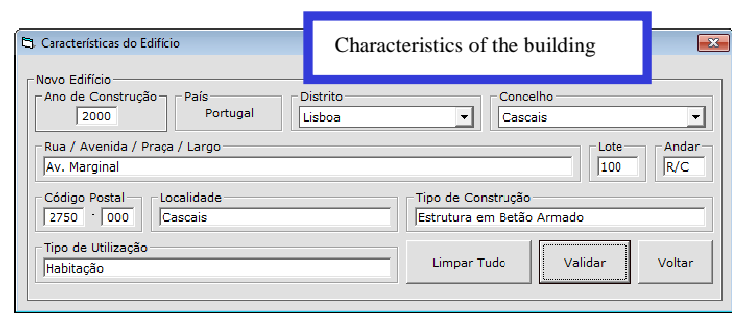

Figure 3. Interface for the detailed description of the building.

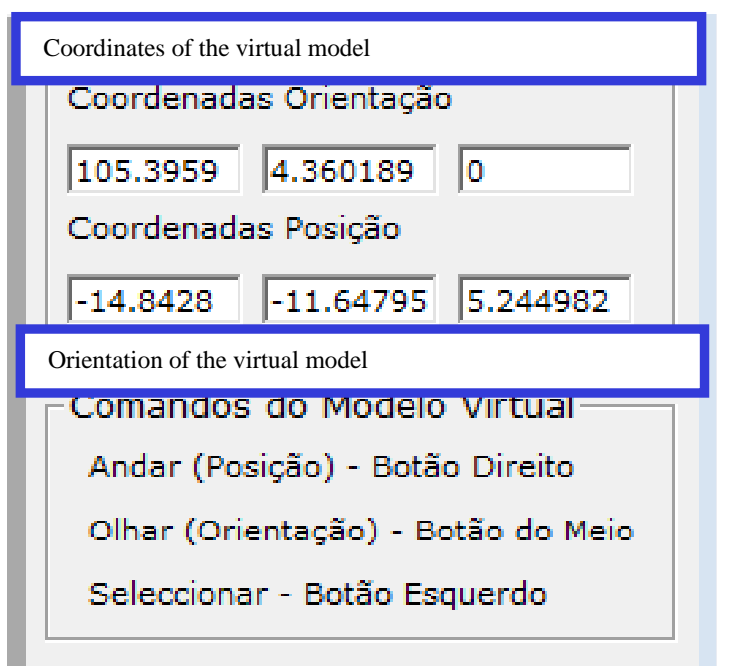

Figure 4. Coordinates and manipulation commands in the virtual model. 


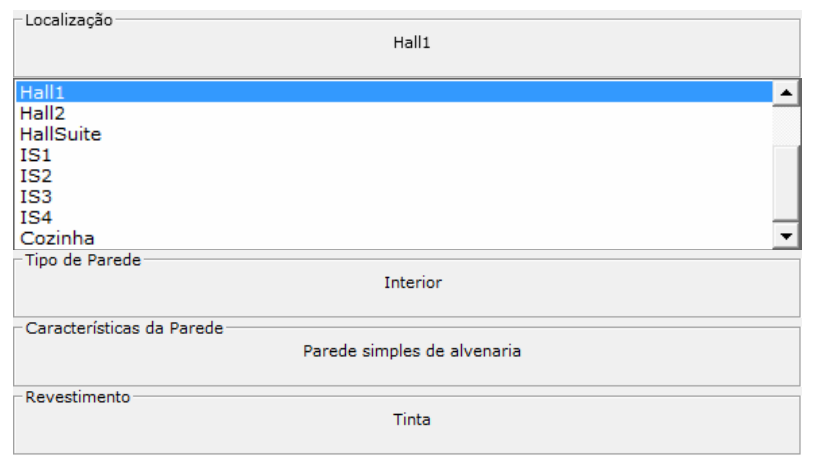

Figure 5. Identification of an element in the virtual model.

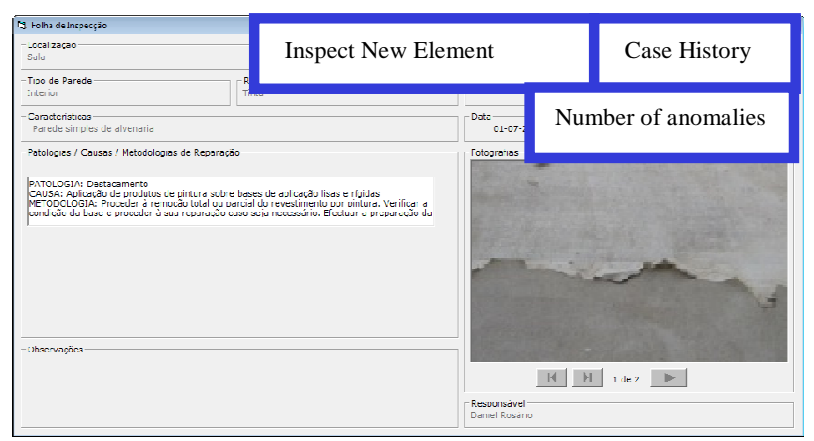

Figure 6. Presentation of the information introduced into the inspection sheet.

Next, using the data base, the irregularity which corresponds to the observed defect, with its probable cause (ageing) and the prescribed repair methodology (removal and repainting) is selected (see highlighted area, Figure 7).

The current size of the pathology should also be indicated since it reveals how serious it is (area of pathology, Figure 7). In the field Observations, the inspector can add any relevant comment (Figure 6), photographs obtained on site can also be inserted into the inspection window and the date of the on-site visit and the ID of the inspector should also be added.

Several different irregularities in the same coating can be analysed (field Number of Pathologies, Figure 6) and other elements can be analysed and recorded and defects observed. Later, the files thus created, associated to each of the virtual model elements, can be consulted (Case History button in the Interface in Figure 6). This same window allows all the data referring to the building and to the completed inspection to be shown, in $p d f$ format (Figure 8).

\subsection{Maintenance Monitoring}

How long the working life of any construction component might be is an estimate and depends on a set of modifying factors related to their inherent characteristics of quality, to the environment in which the building is set

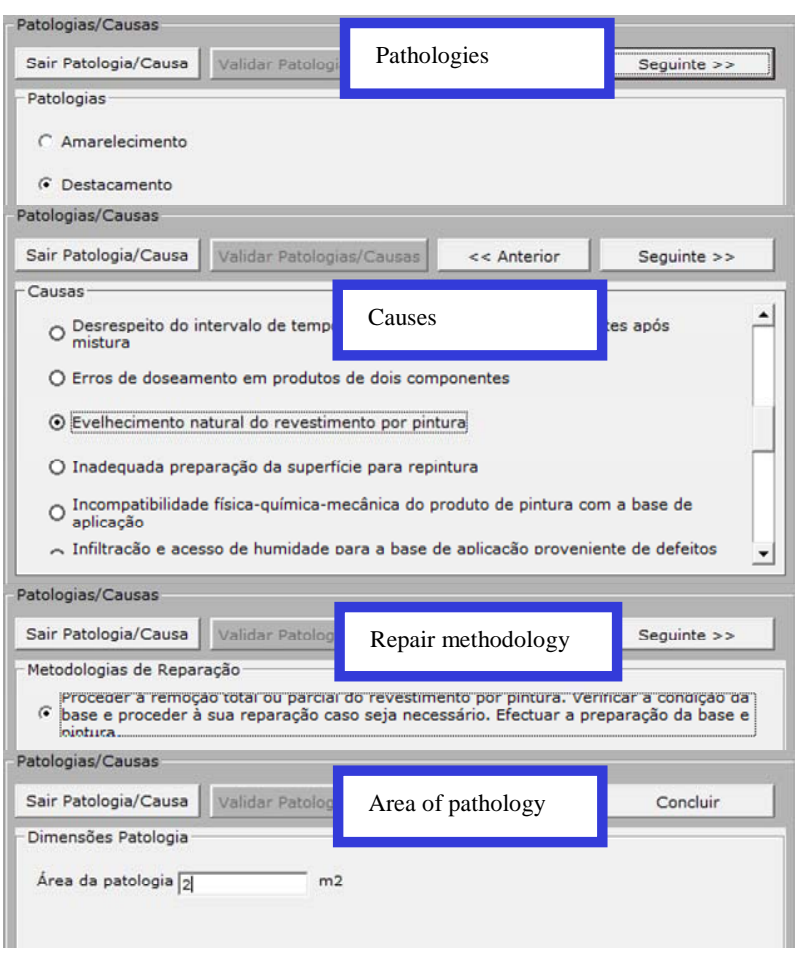

Figure 7. Interface for the selection of the irregularity, probable cause, area and repair methodology.

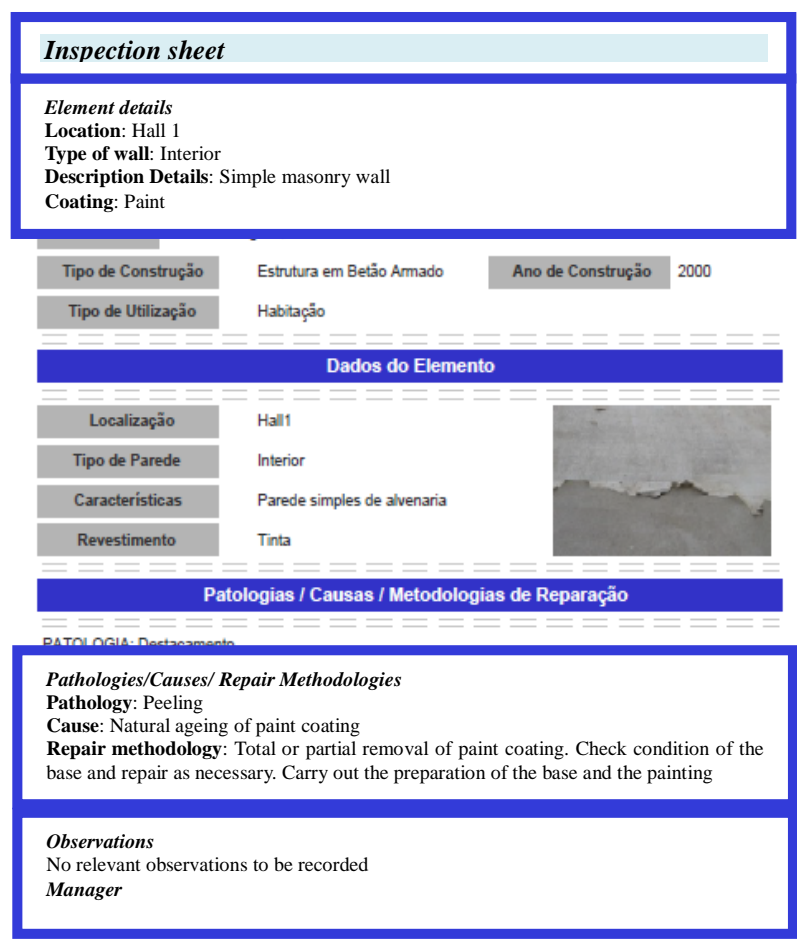

Figure 8. Inspection sheet.

and to its conditions of use [5].

In maintenance strategy planning the probable dates when adverse effects might occur in each of these elements must be foreseen, and the factors which contribute 
to defects must be reduced and their consequences minimized.

The completed model allows the user to monitor the evolution of wear and tear on the paint coating in a house. For this, technical information relative to the reference for the paint used, its durability and the date of its most recent application must be added (Figure 9) to each element through the Maintenance Interface (also accessed from the main interface, Figure 2).

Based on these data, it is possible to link in the date the virtual model is consulted and visualise, in the geometric model, the level of wear and tear as a function of time (see state of repair, Figure 9). The period of time between the date indicated and the date when the paint was applied is compared to the duration advised, in the technical literature, for repainting. The value given for this comparison is associated to the Red, Green, Blue (RGB) parameters which define the colour used for wall in the virtual model (Figure 10).

In this way, the colour visualised on the monitored wall varies according to the period of time calculated, pale green being the colour referring to the date of painting and red indicating that the date the model was consulted coincides with that advised for repainting (Figure 10).

The data for painting and repainting are saved to a list

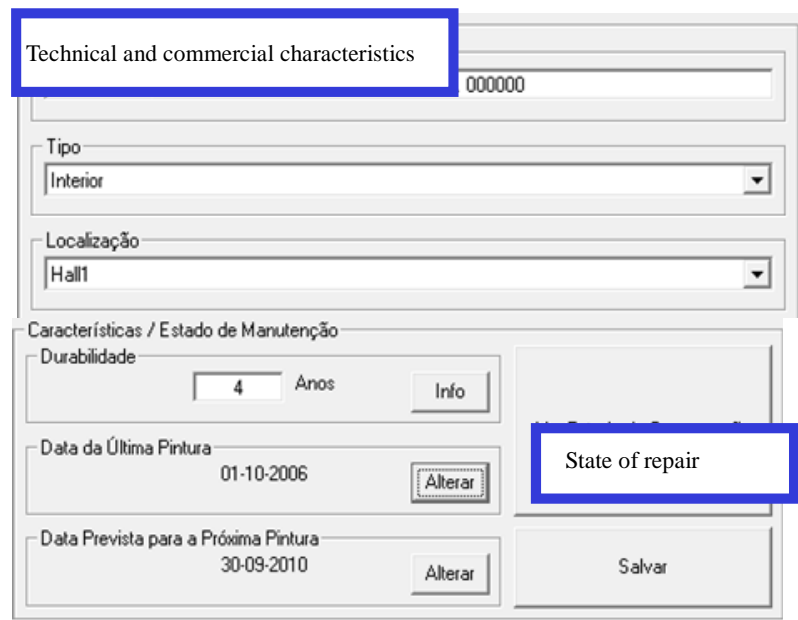

Figure 9. Technical characteristics and the durability of the paint-work.

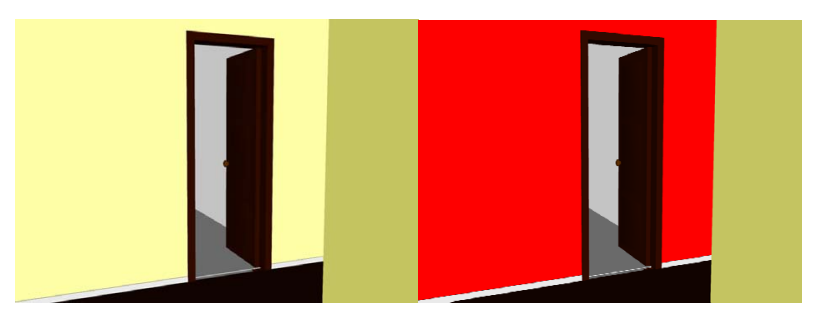

Figure 10. Chromatic alteration of the coating according to its state of deterioration. of coating elements to be monitored in the virtual model (Figure 11). When an element is selected from this list, the corresponding element is represented in the virtual model, through the preview window, in the colour that corresponds to the period of the consultation (Figure 10).

\section{Conclusions}

This application supports the maintenance of painted interior walls and promotes the use of IT tools with advanced graphic and interactive capabilities in order to facilitate and expedite the inspection process. The virtual model, moreover, allows users to see, in the virtual environment, the state of repair of the coating.

The information about pathologies, causes and repair methods, collected from a specialised bibliography, has been organised in such a way as to establish a database to be used as a base for the drawing up of a tool to support building maintenance. The main aim of the application is to facilitate maintenance enabling the rapid and easy identification of irregularities, as well as the possible prediction of their occurrence through the available inspection record. This analysis has been shown as playing an important role in conservation and in the reduction of costs related to the wear and tear of buildings and contributes to the better management of buildings where maintenance is concerned.

In addition to the inspection component, a maintenance component was developed which, being visualized in a VR environment, as well as being highly intuitive, facilitates the analysis of the state of repair of buildings. By means of a chromatic scale applied to the monitored elements, displayed in the walk-through of the geometrically modelled building, it is possible to identify the elements which, predictably, will need timely action. With the possibility of altering the time parameter freely, the user can carry out this analysis either for past instants or for future events, being able, in this way, to forecast future operations. This capacity of the model, therefore, contributes to the avoidance of costs associated to irregularities which, with the passage of time, become more serious and therefore more onerous.

\section{Acknowledgements}

The authors wish to thank the Foundation for Science and Technology for the financial support given for the development of the research project Virtual Reality

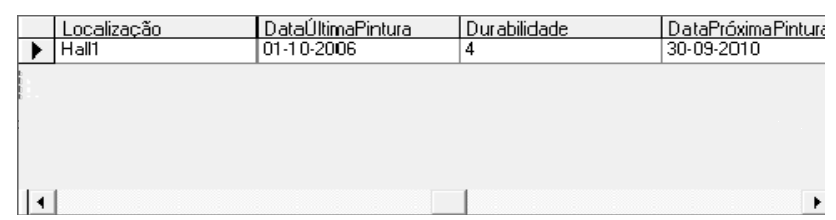

Figure 11. Information relevant to the monitored elements. 
technology applied as a support tool to the planning of construction maintenance. PTDC/ECM/67748/2006 (20082011).

\section{REFERENCES}

[1] M. Eusébio and M. Rodrigues, "Paints, Varnishes and Painted Coatings for Civil Construction,” CS 14, National Laboratory for Civil Engineering, Lisbon, 2009.

[2] C. Lopes, "Anomalies in Painted Exterior Walls: Technic of Inspection and Structural Evaluation,” Construlink Press, Lisbon, 2004.

[3] A. Z. Sampaio and A. Gomes, "Virtual Reality Technology Applied as a Support Tool to the Planning of Construction Maintenance,” Research Project PTDC/ECM/67748, FCT, Lisbon, 2006.

[4] "RGEU-General Regulations for Urban Buildings,” Decree-Law, Lisbon, 1951.

[5] V. Cóias, "Inspections and Essays on Rehabilitation of Buildings,” 2nd Edition, IST Press, Lisbon, 2009, p. 448.

[6] I. F. Collen, "Periodic Inspections in Buildings," Planet CAD Studies, 2003.

http://www.planetacad.com/presentationlayer/Estudo_01. aspx?id=13\&canal _ordem $=0403$

[7] M. B. Farinha, "Construction of Buildings in Practice: Guide Oriented to the Development of Processes and Methodologies of Construction,” Verlag Dashofer, Psicosoma, Lisbon, 2010. http://www.psicosoma.pt/

[8] M. I. Marques, “Durability of Plastic Tint,” ITMC 2, National Laboratory for Civil Engineering, Lisbon, 1985.

[9] A. Moura, "Characteristics and Conservation State of Painted Façades: Study Case in Coimbra," Master Dissertation in Construction, Technical University of Coimbra, Coimbra, 2008.

[10] D. P. Rosário, "Virtual Reality Technology Applied on Building Maintenance: Painted Interior Walls,” Master Dissertation in Construction, Technical University of Lisbon, Lisbon, 2011.

[11] "EON Studio. Introduction to Working in EON Studio," EON Reality, Inc., 2011. http://www.eonreality.com/

[12] “ISTAR - Architectural Laboratory of IST,” Lisbon, 2011. https://fenix.ist.utl.pt/departamentos/decivil/ lateral/o-ecivil/unidades-de-apoio/laboratorios-informati cos 